\title{
Shared Autonomous Vehicles Effect on Vehicle-Km Traveled and Average Trip Duration
}

\author{
Ana T. Moreno (D), Andrzej Michalski, Carlos Llorca (D), and Rolf Moeckel \\ Department of Civil, Geo and Environmental Engineering, Technical University of Munich, Munich 80333, Germany \\ Correspondence should be addressed to Ana T. Moreno; ana.moreno@tum.de
}

Received 7 February 2018; Accepted 18 April 2018; Published 23 May 2018

Academic Editor: Alain Lambert

Copyright (C) 2018 Ana T. Moreno et al. This is an open access article distributed under the Creative Commons Attribution License, which permits unrestricted use, distribution, and reproduction in any medium, provided the original work is properly cited.

\begin{abstract}
Intermediate modes of transport, such as shared vehicles or ride sharing, are starting to increase their market share at the expense of traditional modes of car, public transport, and taxi. In the advent of autonomous vehicles, single occupancy shared vehicles are expected to substitute at least in part private conventional vehicle trips. The objective of this paper is to estimate the impact of shared autonomous vehicles on average trip duration and vehicle-km traveled in a large metropolitan area. A stated preference online survey was designed to gather data on the willingness to use shared autonomous vehicles. Then, commute trips and homebased other trips were generated microscopically for a synthetic population in the greater Munich metropolitan area. Individuals who traveled by auto were selected to switch from a conventional vehicle to a shared autonomous vehicle subject to their willingness to use them. The effect of shared autonomous vehicles on urban mobility was assessed through traffic simulations in MATSim with a varying autonomous taxi fleet size. The results indicated that the total traveled distance increased by up to $8 \%$ after autonomous fleets were introduced. Current travel demand can still be satisfied with an acceptable waiting time when 10 conventional vehicles are replaced with 4 shared autonomous vehicles.
\end{abstract}

\section{Introduction}

Autonomous vehicles (AVs), or driverless cars, have the potential to make a revolution on travel behavior and urban development. While AVs might reduce traffic congestion by improving network capacity and traffic efficiency, they could also increase traffic volumes due to induced travel demand because of improved convenience of travel or because of empty trips of shared autonomous vehicles to pick up the next passenger or to find a parking place $[1,2]$. Traditional modes of car, public transport, and taxi are slowly beginning to lose market share to intermediate modes such as shared taxis (e.g., Cabify, Lyft, or Uber) or shared vehicles (e.g., Car2Go, DriveNow, or ZipCar) [3]. When shared AVs (SAVs) are used individually (no ride sharing), they can be assimilated as autonomous taxis. In this sense, there has been a growing interest in how SAVs can operate in urban areas and how they would affect urban mobility.

City-wide simulations of complete replacement of private conventional vehicles (PCVs) with SAVs in Berlin, Lisbon, and Austin indicated that one SAV could replace the demand served by ten PCVs [4-6]. In a region-wide study for the greater Munich metropolitan area, the substitution rates were at least equal to 3 -for-10 to maintain reasonable waiting times. In that study, AT use was randomly selected at predefined penetration rates of $20 \%$ and $40 \%$ [7]. Because of empty trips, vehicle-kilometers traveled (VKT) increased between $4 \%$ and $51 \%$, depending on the penetration rate of ATs and the case study.

Previous studies on the impact of SAVs on urban mobility had to simplify various assumptions, such as penetration rates, willingness to use SAVs, or limiting the study area. Milakis et al. [8] expected that the percentage of AVs in vehicle fleets will be less than $11 \%$ in 2030 and between $7 \%$ and $61 \%$ in 2050 . Therefore, conventional and autonomous vehicles are probably going to coexist in the next few decades. To forecast the demand side, penetration rates should consider the willingness to use the technology as shared vehicle (single occupancy or ride sharing). Additionally, limiting the study area to single cities can be a significant shortcoming in polycentric regions, particularly for commute trips and longdistance trips. 
The objective of this paper is to estimate the impact of SAVs on average trip duration and VKT in the greater Munich metropolitan area, taking into account the willingness to use SAVs.

The organization of the paper is as follows. Section 2 reviews research related to regional impacts of AVs and subjective perceptions by users, after which Section 3 presents the methodology. Results and analyses are discussed in Section 4. Finally, conclusions are presented in Section 5.

\section{Literature Review}

Researchers have developed frameworks for managing SAVs fleets and their daily operations [9-11] or determined the driving forces for their implementation in large cities [12]. Only rarely it has been attempted to model travel behavior with SAVs at a regional scale. Notable exceptions are the transport analyses in Berlin [4], Lisbon [6, 13], downtown Austin [5], and the metropolitan area of Munich [7] and the optimization of the New York City taxicab fleet using SAV real-time ride sharing [14]. On the demand side, some studies had modeled acceptance and use of SAVs [15-18], even though many others had used stated preference surveys to evaluate the willingness to pay extra for privately owned AVs, as summarized by Becker and Axhausen [19].

2.1. Effects of Shared Autonomous Vehicles on Urban Mobility. Bischoff and Maciejewski [4] tested a complete fleet of shared autonomous vehicles in Berlin using simulations in MATSim with the extension RoboTaxi. MATSim is a multiagent transport simulation software that allows performing largescale agent-based transport simulations. The model was run for a typical weekday in Berlin 2008 assuming the modal split between auto and transit. The scenarios considered a $100 \%$ penetration rate of shared autonomous vehicles. The service assumption was no advance bookings and individual rides (no ride sharing). The initial fleet was distributed in the morning proportionally to population density. The results indicate that waiting times were distributed differently in the city center and the outskirts. While waiting times were quite stable during the day in the city center, some regions had average waiting times of almost 20 minutes. Those regions also had an overall low share of trips and longer pickup distances (from $1 \mathrm{~km}$ to $5 \mathrm{~km}$ ) and produced an empty ride share of up to $45 \%$. The results indicate that $1 \mathrm{SAV}$ can serve the same demand as 11 PCVs (substitution rate of 1-for11).

Fagnant and Kockelman [5] simulated one typical day in downtown Austin in 2010 using MATSim. All personal trips were dispatched using PCVs. Departure times were distributed using Seattle's household travel diaries. Average travel speeds across the network by hour were obtained from MATSim. An independent module was used to model SAVs, including their trip assignment, movement, relocation, and initial fleet generation. They considered single occupancy and ride sharing, with an increase of travel time by $20 \%$ and $40 \%$. Ride sharing reduced the number of extra VKTs from $8.7 \%$ to $1.5 \%$ due to less and shorter empty trips. The percentage of shared miles increased from $4.8 \%$ to $11.2 \%$ after allowing
$40 \%$ longer travel times. Average waiting time was lower than 2 minutes with a substitution rate of 1 -for- 10 .

The International Transport Forum at the OECD [6] simulated the synthetic trips of one average day in Lisbon. All trips with distances below $1 \mathrm{~km}$ were assumed to be traveled by foot or bicycle ( $18 \%$ of trips), while trips with origins and destinations close to metro stations were directly assigned to metro (22\% of trips). Auto trips were partially substituted by single occupancy SAVs and ride sharing SAVs. They tested nine scenarios varying the mode of AV technology (single occupancy or ride sharing), penetration rate $(50 \%$ or $100 \%$ of AV technology), presence of metro (yes or no), and the base scenario with $100 \%$ of PCVs. Fleet size was assessed by calculating the required number of AVs to deliver the same level of mobility as with private conventional cars in terms of travel origins, destinations, and length of trip. Substitution rates 1 -for-10 and 2-for-10 were obtained for the scenario of $100 \%$ SAVs and $50 \%$ SAVs, respectively. Travel volumes increased between $6 \%$ and 51\%, depending on the AV technology and the penetration rate. For $50 \%$ of penetration rate, VKTs increased by $31 \%$ with ride sharing and $51 \%$ with single occupancy. The impact on v/c ratio was little, from an average of $42 \%$ on the base scenario to $45 \%$ on the worst case. Finally, average waiting times were between 3 and 4 minutes.

In a follow-up paper, Martinez and Viegas [13] examined the changes in mobility and $\mathrm{CO} 2$ emissions in Lisbon in a scenario where taxi-buses and shared taxis modes replace private conventional cars, buses, and taxis. Metro and walking are kept as modes. The trip origins and destinations are the same as in the previous study [6]. For the current scenario mode choice, they derived a nested-logit model based on age and income, land use and mode availability, and transport operation attributes. For the future scenario, the mode choice process had sequential rules due to the absence of stated preferences for shared mobility solutions. Walking, metro, shared-taxi, and taxi-bus selection was tested step by step. Users selected taxi-bus only if they had not selected the previous options. Taxi-buses should be requested 30 minutes in advance, while shared taxis had real-time booking system and performed door-to-door services. If taxi-buses could not supply the demand in a reasonable waiting time, users were upgraded to shared-taxi. The results from one average day indicate that carbon emissions and VKTs would be reduced by $40 \%$ and $30 \%$, respectively. The substitution rate from current private conventional vehicles to AVs fleet was 3-for100 to produce the same mobility, showing an impressive reduction.

Llorca et al. [7] simulated the use of SAVs to substitute partially the travel demand for commute trips in the greater Munich metropolitan area. Commute trips were generated based on the synthetic population. A two-step mode choice model was applied. First, individuals selected among current transport modes: auto, public transport, bicycle, and walking. Secondly, individuals that selected auto mode opted to change their private conventional vehicle by a SAV. This selection was random and the threshold was equal to the predefined penetration rate. Eleven scenarios were considered varying penetration rate $(0,20$, and $40 \%)$ and substitution rate 
(1-for-10 to 5 -for-10). The results from the simulations in MATSim showed that the total VKTs increased up to $5 \%$ and $10 \%$, for penetration rates of 40 and $20 \%$, respectively. Moreover, the presence of SAVs increased not only SAV average trip duration due to waiting times, but also PCV average trip duration due to SAV empty trips. They suggested substitution rates of 3 -for-10 to provide waiting time shorter than 8 minutes.

2.2. Autonomous Vehicle Acceptance and Use. Zmud and Sener [15] and Bansal et al. [16] conducted two state preference online surveys in the Austin metropolitan area. The objective was to gather how likely people are to use AVs, which factors influence their willingness to use them, and how that might change travel behavior in the future. They gathered 556 and 347 responses online, respectively. Zmud and Sener [15] concluded that personality and psychology of the individual, specially self-perception of risk and usage of new technologies, are currently much more important than the demographic characteristics. On the other hand, Bansal et al. [16] found statistical significance among demographic characteristics. Specifically, their results indicate that fulltime male workers living in urban areas, without children, are more likely to use shared AVs more frequently. Holding a driver license decreased the likelihood of using shared AVs.

On follow-up stage, Zmud and Sener conducted face-toface interviews with 44 persons to assess the perceptions of self-driving and their potential usage of AVs. They indicate that "the intent to use is an importance concept because the technology is not yet on the market. It is not until the product becomes tangible and drivers have an opportunity to experience it "for real" that they can form judgments and provide reliable and valid responses to questions pertaining to actual use". Most of the 44 respondents would rather use autonomous vehicles as private cars (59\%), compared to $41 \%$ that would use them as shared vehicle. There were no age or income difference among individuals interested in shared vehicles. Additionally, $66 \%$ of the respondents indicated that their annual mileage would not change with AVs.

Krueger et al. [17] identified the characteristics of users who are likely to adopt shared AVs using a stated choice online survey. The respondents were asked to indicate whether they would switch to a SAV on a trip they recently undertook. A total of 435 residents in major metropolitan areas of Australia completed the survey. Individuals that use all four modes relatively frequently, as well as individuals that travel by car, public transport, and walk frequently are more likely to use shared AVs. Moreover, the willingness to use SAVs was higher for shopping and medical trips. Demographic characteristics were not statistically significant.

Finally, Haboucha et al. [18] conducted a stated preference survey in Israel and North America. The online survey gathered 721 responses. They concluded that only $75 \%$ of individuals would be willing to use SAVs even if the service was completely free. Among their sociodemographic characteristics, young males with higher education will be more likely to use SAVs.

\section{Materials and Methods}

3.1. Study Area. The study area was the greater Munich metropolitan area. It included the cities of Munich, Augsburg, Ingolstadt, Landshut, and Rosenheim and their respective suburbs to cover the large commuting shed of the Munich region (Figure 1). The study area covers 150 by $150 \mathrm{~km}$. The delineation of the study area was carried out using the share of commuters' flow into central cities and among cities. This metropolitan area includes a population of 4.5 million living in 2.1 million households and 14 million person-trips every day.

After delineating the study area, the municipalities were divided into traffic analysis zones (TAZs) using a gradual raster-based zone system [20]. The total number of TAZs was 4,950 , and the finest resolution was 200 by $200 \mathrm{~m}$. The zone system nested TAZs within the municipal regions respecting municipal boundaries, which allowed allocating the population according to the census division (Figure 2).

3.2. Stated Preference Online Survey. Stated preferences devoted to AV technology were gathered through an online survey. A 19-question survey was created in Google Forms and placed on the research group website. It was designed in four main parts:

(1) Description of AVs and General Knowledge about the Technology. Individuals were asked about how informed they were about the development of AVs and how they subjectively perceived the spread of information about AVs.

(2) Travel Behavior Characteristics on an Average Day. Individuals reported their number of trips (from one origin to one destination), the used modes of transport, the most important mode of transport, how much time they spent traveling daily, and how safe they perceive conventional vehicles and autonomous vehicles.

(3) Willingness to Use AVs. Individuals selected which modes they would consider using AVs (multiple choice), for which situations they could imagine using AVs (multiple choice), which increase on travel time they would be willing to accept with AVs, if they would consider purchasing a private AV (scale), and how much extra they would be willing to pay to own one.

(4) Sociodemographic Profile. Individuals provided their age range, gender, household size, household income, car ownership, and county of residence.

The average time to respond to the survey was below 10 minutes. The survey was offered in both German and English. The survey targeted respondents in the Munich metropolitan area. The link to the survey was posted at the research group website, website of the city of Fürstenfeldbruck and the monthly newsletter of Fürstenfeldbruck, Facebook group pages for Munich-region activities, and MotorForum website in Munich.

The survey was available online for six weeks along March and April 2017 and gathered 86 responses. In order to 


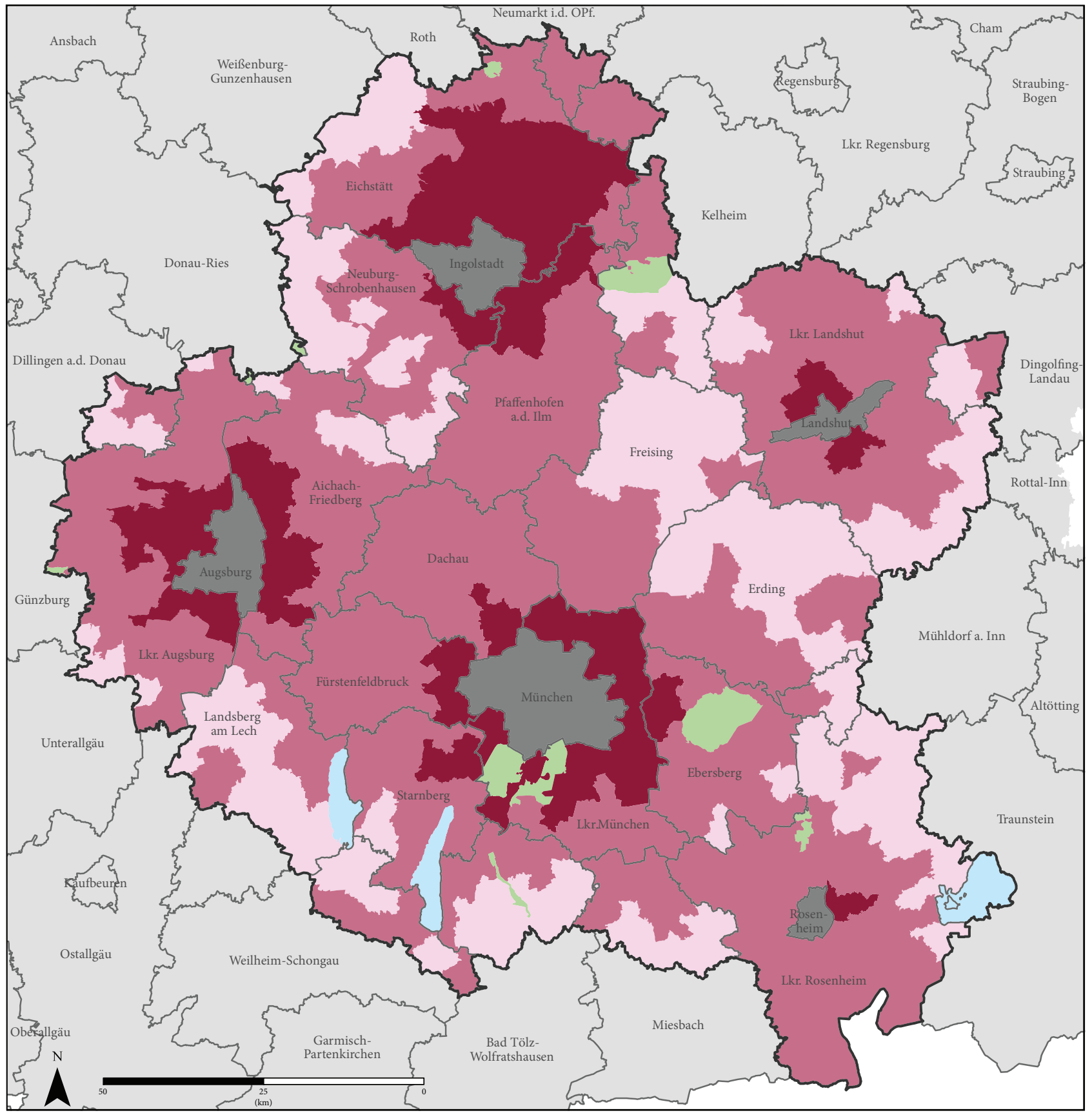

Percentage of commuters in the core region out of total commuters per municipality

Percentage $<25 \%$ or outside of the study area $<25,0$

Figure 1: Delineation of the Greater Munich metropolitan area.

increase the sample, 20 on-street surveys were conducted in Fürstenfeldbruck with the same questions as on the online survey. The total sample was 106. English- and Germanspeaking respondents were evenly distributed, and around $70 \%$ of respondents were men. $98 \%$ of English-speaking respondents were younger than 35 years old and only $19 \%$ owned a car. Conversely, 62\% of German-speaking respondents owned a car and covered a wider spectrum of age (18-65 years old).

3.3. Travel Demand. Travel demand was generated based on the synthetic population of the land use model SILO [21, 


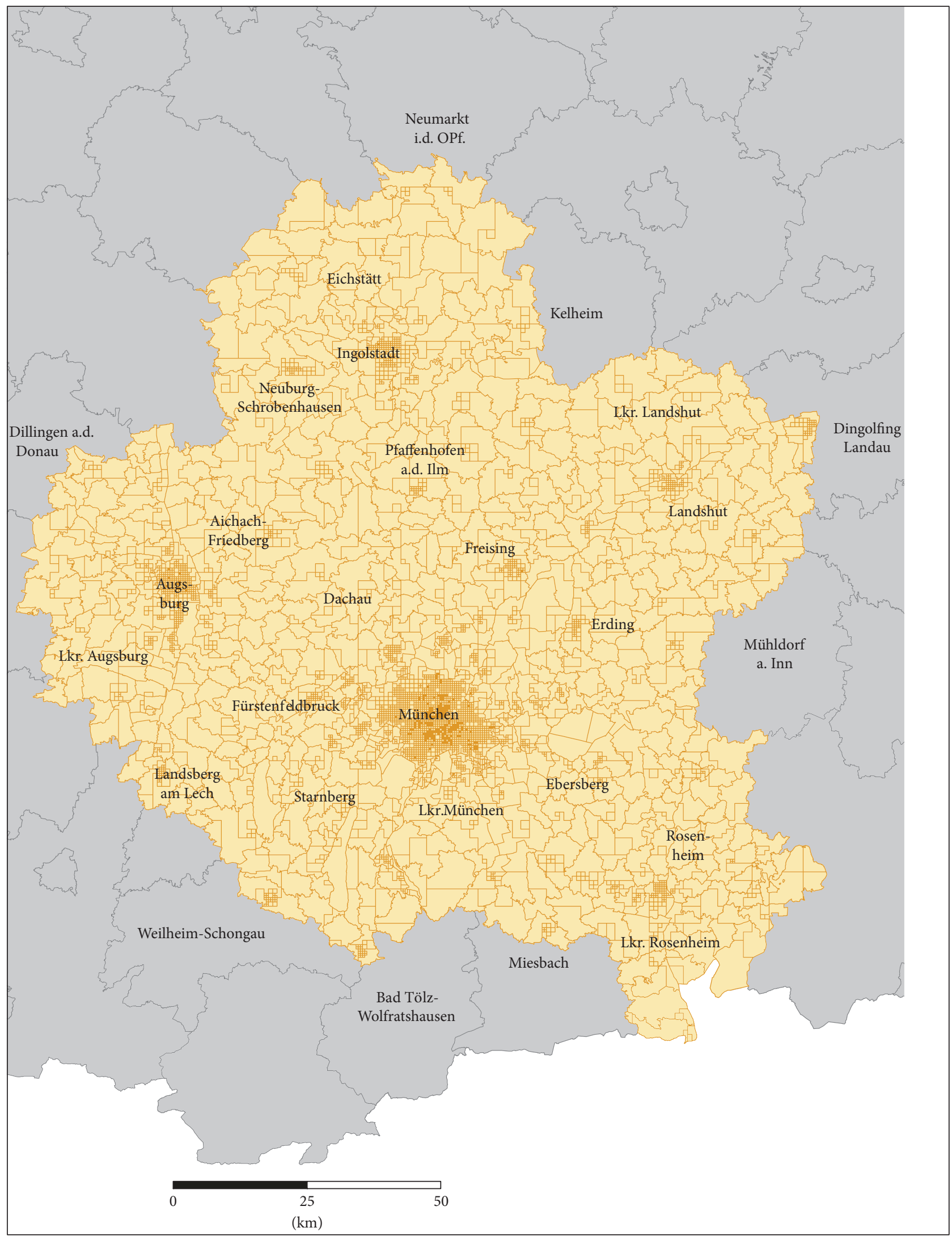

FIGURE 2: Greater Munich metropolitan area: counties and traffic analysis zones. 


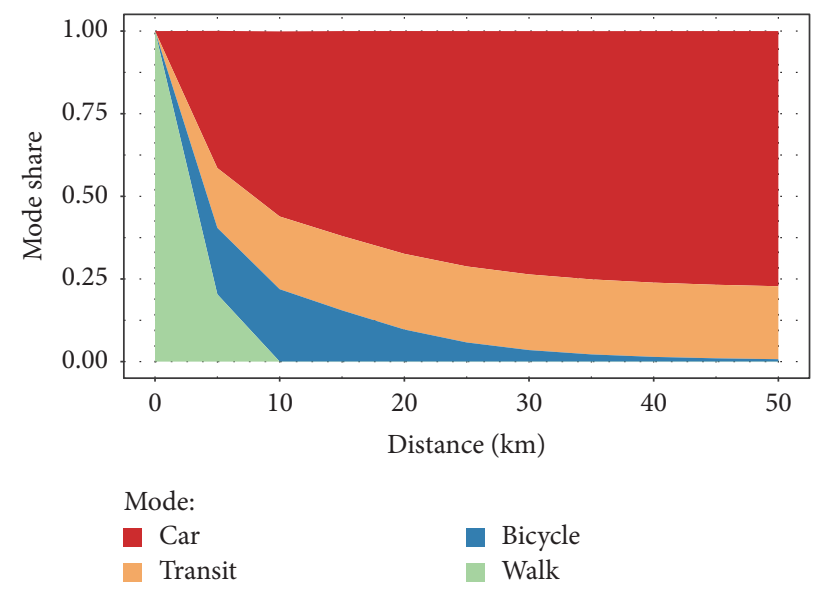

Figure 3: Mode share by distance: first step.

22]. The synthetic population is a microscopic representation of the 4.5 million people living in this study area. It was created combining a sample of disaggregate members of a population (microdata) in a way as to match key distributions for the entire population. Based on the available data in the control totals and microdata, we selected 59 control attributes: 46 attributes at the municipality level and 13 attributes at the county level. The attributes included age by gender, occupation by gender, nationality, household size, tenure status, dwelling living space, or dwelling type (http://www.silo.zone). For the optimization phase, we used Iterative Proportional Updating procedure, while Monte Carlo sampling was selected for the allocation phase. Households were allocated at the TAZ level, as well as jobs. Because the assignment model MATSim can work with micro locations as trip ends, households and jobs were randomly assigned to one point within their corresponding TAZ instead of the TAZ centroid.

For this exercise, we sent all workers to their workplace and all tertiary students to their educational facility during the morning hours. A destination choice model was calibrated to assign workplaces to workers and schools to students. To validate the model, we used home-basedwork (HBW) and home-based-education (HBE) trip length frequency distributions from the German Household Travel Survey-Mobilitaet in Deutschland (MiD 2008) [23]. Homebased-other $(\mathrm{HBO})$ trips were also generated using $\mathrm{MiD}$ observed trip rates and trip length frequency for HBO trips. Time of day depended on the job type and was randomly assigned for $\mathrm{HBO}$ trips.

Mode was selected in two steps. At the first step, the available modes were four: auto; public transport (metro, tram, and bus); bicycle; and walk. Mode was selected according to distance, with short trips being dominated by nonmotorized modes and the longest trips being dominated by auto (Figure 3 ).

At the second step, auto users selected whether to travel with private PCV or one SAV. The probability of one individual to select SAV was predicted using the logit model from the stated preference survey. The logit model will be defined in Section 4.1.
3.4. Traffic Simulations in MATSim. Once travel demand is generated, traffic assignment was carried out using the agent-based simulator MATSim [24]. The road network for the entire study area was downloaded from the Open Street Map service. The network contains 138,000 links and 68,000 nodes. The total number of agents was scaled to $5 \%$ to complete the simulation in reasonable runtimes [7, 25]. MATSim capacity factor and storage factor parameters were set up to 0.10 and 0.18 , respectively, based on previous research recommendations. The simulation of a fleet of shared autonomous vehicles was based on the MATSim extensions DVRP (Dynamic Vehicle Routing Problem) [25] and RoboTaxi [4]. These extensions optimize the use of a fleet of autonomous taxis to serve a certain demand of trips, based on a demand-supply balancing strategy. Under peak hour demands, this optimization strategy maximizes the use of vehicles to reduce waiting times instead of serving the taxi request as they appear.

Using these extensions, shared autonomous vehicles' trips were always done for individual passengers. Input data for the simulation of SAVs are the number of SAVs and their initial location. Initially, shared autonomous vehicles were distributed randomly over the entire network, proportionally to the density of links.

A total of 11 scenarios were simulated to analyze the effect of different substitution rates (Table 1). Penetration rate describes the share of trips made by SAVs, while substitution rate assumes that vehicles are not personally owned but shared autonomous vehicles. The substitution rate describes how many PCVs can be replaced by one SAV. If every household owning a PCV replaces the car with a personally owned SAV, the substitution rate would be 10 -for-10. If-as suggested by scenarios in Berlin [4] and Lisbon [6] —one SAV replaces ten PCVs, the substitution rate would be 1-for-10.

\section{Results and Discussion}

4.1. Willingness to Use Shared Autonomous Vehicles. In order to assess the willingness to use SAVs, we analyzed in detail the responses to the multiple choice question "How would you consider using automated vehicles?". The question had 8 possible answers:

(1) As my own private car.

(2) As a shared car-individual use (e.g., Car2Go, DriveNow).

(3) As a shared car-shared used with other passengers (ride sharing).

(4) As a rental car.

(5) As a taxi (e.g., Uber, Cabify).

(6) As a delivery service.

(7) I won't use them.

(8) Other use.

Individuals that selected either AVs as taxi and/or shared vehicle-individual use were considered willing to use SAVs. 
TABLE 1: Simulated scenarios (agents, trips, and vehicles already scaled to 5\%).

\begin{tabular}{|c|c|c|c|c|c|c|}
\hline Scenario & Substitution rate $^{1}$ & SAVs & Penetration rate $^{2}$ & PCV trips & AV trips & Number of agents \\
\hline 0 - base & No SAVs & 0 & 0 & 62,177 & 0 & 53,000 \\
\hline 1 & 1-for-10 & 1,523 & \multirow{10}{*}{$24.5 \%$} & \multirow{10}{*}{46,941} & \multirow{10}{*}{15,236} & \multirow{10}{*}{53,000} \\
\hline 2 & 2 -for-10 & 1,692 & & & & \\
\hline 3 & 3 -for-10 & 1,904 & & & & \\
\hline 4 & 4 -for-10 & 2,176 & & & & \\
\hline 5 & 5 -for-10 & 2,539 & & & & \\
\hline 6 & 6 -for-10 & 3,048 & & & & \\
\hline 7 & 7-for-10 & 3,809 & & & & \\
\hline 8 & 8 -for-10 & 5,078 & & & & \\
\hline 9 & 9 -for-10 & 7,618 & & & & \\
\hline 10 & 10 -for-10 & 15,236 & & & & \\
\hline
\end{tabular}

TABLE 2: Logit model for willingness to use shared autonomous vehicles.

\begin{tabular}{|c|c|c|c|c|c|}
\hline Coefficients & Estimate & Error & $t$-value & $\operatorname{Pr}(>|t|)$ & Significance \\
\hline Intercept & -3.4893 & 0.82455 & -4.2317 & 0.000023 & $* * *$ \\
\hline Male & 1.2622 & 0.52426 & 2.4076 & 0.016058 & * \\
\hline Female & (Reference) & & & & \\
\hline 3-4 trips per day & 1.5181 & 0.46973 & 3.2319 & 0.001230 & ** \\
\hline $0-2$ or $4+$ trips per day & (Reference) & & & & \\
\hline$<35$ years \& owns car & 2.2993 & 0.76888 & 2.9904 & 0.002786 & $* *$ \\
\hline$<35$ years \& does not own car & 1.5892 & 0.66211 & 2.4003 & 0.016384 & * \\
\hline$>35$ years \& does not own car & 1.8386 & 1.01506 & 1.8113 & 0.070097 & . \\
\hline$>35$ years \& owns car & (Reference) & & & & \\
\hline
\end{tabular}

Note. Signif. codes: $0^{* * *} 0.001^{* *} 0.01^{*} 0.05^{*} 0.1$; log-likelihood: -57.709 ; McFadden $R^{2}: 0.19779$; likelihood ratio test: chisq $=28.458(p$ value $=2.9618 \mathrm{e}-05)$.

$41.5 \%$ of the respondents were willing to use AVs as SAVs, while $58.5 \%$ were not willing to use them as SAVs.

A logit model was applied to estimate the willingness to use autonomous vehicles as SAVs. We used the mlogit package in $R$ [26]. Age and language had a correlation higher than 0.5 and therefore were not included simultaneously on the statistical analysis. No other pair of variables showed correlations higher than 0.5 . The tested independent variables were age, gender, household size, number of trips per day, time spent traveling per day, car ownership, main transport mode, and the correlations among variables. Stepwise selection was followed to estimate the model with only significant variables, at the $90 \%$ level of confidence. Table 2 shows the summary of the final model, with McFadden $R^{2}$ equal to $19.8 \%$.

The statistically significant variables are gender, number of trips per day, and the correlation between age and car ownership. Males are more likely to travel with SAVs than females. The least prone to travel with SAVs are individuals older than 35 years that own a conventional car. Other age groups and car ownership status show higher probability of using SAVs, being the most likely to use SAVs individuals younger than 35 years that own a conventional car. In this sense, young car owners will be more willing to experiment with new driving options. Finally, individuals that travel between 3 and 4 trips per day are more likely to use SAVs than less or more mobile individuals. It is expected that individuals that engage in more trips per day relay on a single mode of transport more available or reliable, while less mobile individuals can be hesitant to change their mode of transport. The results agree with previous research $[16,18]$.

Table 3 shows the resulting modal split on the base scenario without SAVs and the remaining scenarios with SAVs, by trip purpose. The modal split on the base scenario was calibrated using MiD 2008 data. Half of working and educational trips (HBW, HBE) are made by auto, while $18.6 \%$ are made in public transport. For HBO trips, most of them rely on nonmotorized modes (71.5\%), given the shorter trip lengths. In all trip purposes, the share of SAVs on the auto mode is $26.3 \%$.

4.2. Effect of SAVs on Average Trip Duration and VMTs. Average trip duration was compared across different scenarios, individually for SAVs and PCVs, and for all the vehicles (Figure 4). The trip duration was the sum of the driving time and waiting time (with the latter being 0 for PCV trips). Average trip duration of SAV trips was strongly related to the substitution rate, with very long trips (caused by longer wait times) when it was 3-for-10 or lower. With higher substitution rates than 3-for-10, average trip duration decreased at a constant rate. For substitution rates higher than 8 -for-10, the average trip duration of SAVs was very close to PCVs. When 
TABLE 3: Modal split by trip purpose and scenario.

\begin{tabular}{|c|c|c|c|c|}
\hline \multirow{2}{*}{ Mode } & \multicolumn{2}{|c|}{$\mathrm{HBW}+\mathrm{HBE}$} & \multicolumn{2}{|c|}{$\mathrm{HBO}$} \\
\hline & Without SAVs & With SAVs & Without SAVs & With SAVs \\
\hline Private conventional vehicle (\%) & 51.1 & 37.7 & 20.7 & 16.1 \\
\hline Shared autonomous vehicles (\%) & - & 13.4 & - & 4.6 \\
\hline Public transport (\%) & 18.6 & 18.6 & 7.8 & 7.8 \\
\hline Bicycle (\%) & 13.2 & 13.2 & 6.5 & 6.5 \\
\hline Walk (\%) & 17.1 & 17.1 & 65.0 & 65.0 \\
\hline Total (\%) & 100 & 100 & 100 & 100 \\
\hline
\end{tabular}

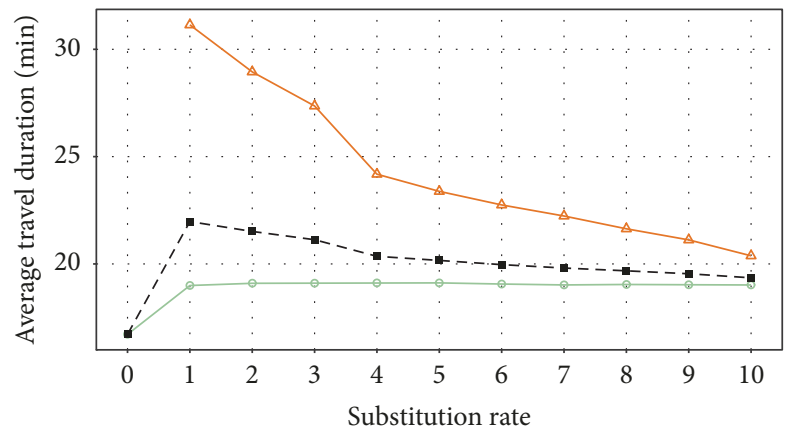

$$
\begin{aligned}
& \text { Mode } \\
& -\quad \text { CV } \\
& \triangle \text { AT } \\
& -- \text { All }
\end{aligned}
$$

FIGURE 4: Average travel time by mode depending on substitution rate.

the substitution rate was 3 -for-10, the average waiting time for SAV travelers was around $8 \mathrm{~min}$, and it was less than 5 minutes for substitution rates of 4 -for- 10 or higher.

The presence of SAVs triggered an increase of the average trip duration of PCVs. The increase (of up to a $14 \%$ with respect to the base scenario, from 16.7 to 19.0 minutes) was caused by additional traffic related to empty trips. The increase of trip duration was slightly higher when the substitution rate of AV trips was between 3-for-10 and 6-for10: for higher substitution rates, the amount of empty trips is smaller while, for smaller substitution rates, the amount of vehicles in the network is smaller.

As a result of increasing both average travel times of SAVs and PCVs, the average trip duration of all modes also increased. As can be observed in Figure 2, the presence of SAVs showed in all scenarios an increase of the average trip duration, caused by increased congestion and added waiting times for SAVs. When the substitution rate was low (1-for10 ), the average trip duration of all trips increased $31 \%$, from 16.7 minutes to 22.0 minutes. For substitution rate of 3 -for- 10 , suggested by Llorca et al. [7] for the Munich region, it still had an increase on average trip duration of $21 \%$, from 16.7 to 21.1 minutes.

It is unknown territory how we will perceive "on the average" the waiting time for a SAV. While in some situations people could spend waiting time doing other things (such as preparing for the trip at home), the same person could face another situation on which waiting time is bothersome (such
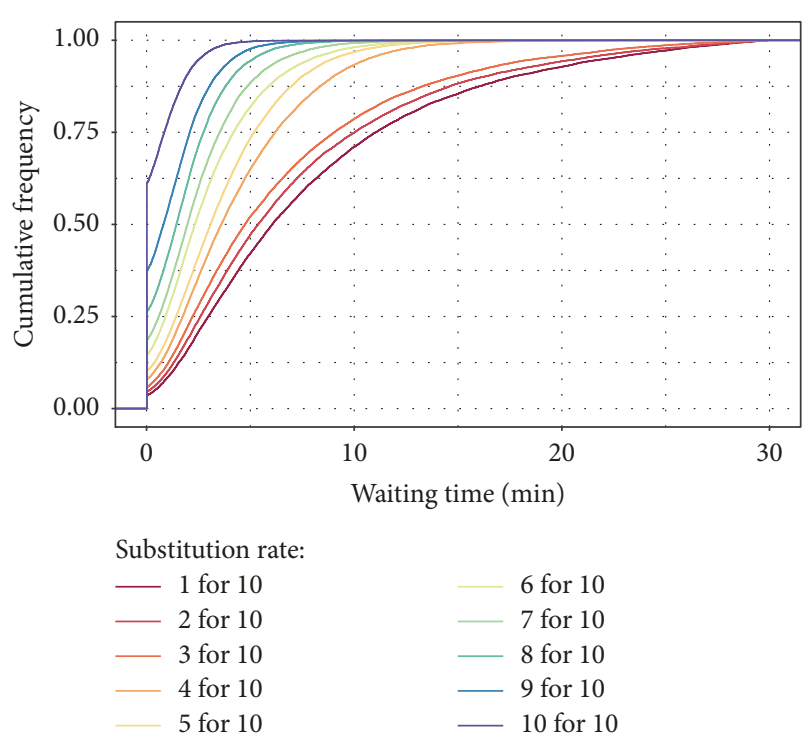

FIGURE 5: Waiting time cumulative distribution function by substitution rate.

as finishing a doctor's appointment and wanting to be picked up). In this sense, average waiting time would not be the most appropriate performance measure. Waiting time cumulative distribution function could be used instead (Figure 5). It indicates the percentage of users served within a particular waiting time, similar to headways in public transit lines. As observed, substitution rates lower than 3-for-10 provided longer waiting times which ranged between 0 and 30 minutes. For substitution rates of 4 -for-10 or higher, the range of waiting times is between 0 and 10 minutes with a confidence of $95 \%$. It implicates that the maximum waiting time is lower than 10 minutes, providing a more reliable service.

Figure 6 shows that all the scenarios with SAV involved an increase of the VKT with respect to the base scenario. The results showed an increase of the total driven kilometers in the entire area in all the scenarios with AVs because of empty trips. The maximum increase was found for the lowest substitution rates: $8 \%$ increase for 1 -for-10 and $7 \%$ increase for 3 -for-10. This increase drops for substitution rates below 4 -for- 10 and is less than $4 \%$.

The results agree with Fagnant and Kockelman [5] findings, where extra VKT was around $8.7 \%$ for single occupancy SAVs, and are smaller than the estimates for Lisbon of extra 51\% VKT [6]. Nevertheless, they are greater than the 


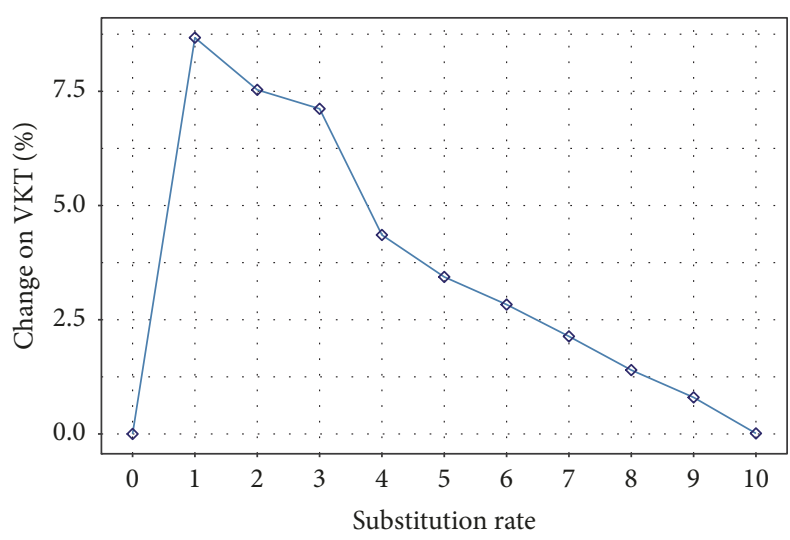

FIGURE 6: Change on VKTs with respect to base scenario depending on substitution rate.

estimates for the Munich region with $20 \%$ of penetration rate (less than 5\% extra VKT). It could be because previous SAVs users were randomly distributed along the network but now are more clustered due to their travel behavior. Therefore, the same amount of trips by SAV could require longer empty trips. In this sense, ride sharing would be more efficient to reduce extra VKTs in our study area.

\section{Conclusions}

The results and conclusions are plausible within the simulation range and for the considered assumptions. In this research, we assumed that the willingness to use AVs as SAVs is a proxy of the actual use. It is readily admitted that the current lack of experience to travel in an AV limits the reliability and validity of survey respondent on the actual use of AVs. The limited sample of the survey is likely to have introduced a bias to the results. However, those surveys help making modeling assumptions less arbitrary when AVs need to be analyzed in transport modeling. To prepare cities to the upcoming arrival of AVs, transport models need to be built today to analyze the impact of this technology. Given the lack of observed data, surveys in conjunction with travel behavior theory is the most promising approach to reasonable model in the unknown future.

The described implementation makes the simplifying assumption that travel demand remained unchanged after AVs were introduced. Given the fundamental change AVs provide to the transport system, this is rather unlikely. Mobility-as-a-Service (MaaS) provider claims that transportation in shared AVs will be less expensive than current MaaS rates. A transport option that is cheaper and more convenient is likely to increase in demand. This induced demand generated by AVs has not been quantified with evidence yet. Transportation planners need to be aware that induced demand will make it rather unlikely that AVs will solve congestion problems. Transportation modelers need to find ways to explicitly model suppressed and induced travel demand [27].

It is planned to further improve the simulation of AVs by integrating the described model with a microscopic travel demand model and a land use model. The microscopic travel demand model MITO simulates travel behavior individually for every household $[28,29]$. It is planned to model not only the activity, for which a certain trip is conducted, but also the activity done while traveling. This is relevant for transit travelers already today, as someone might do some work while commuting and thereby shorten the hours spent in the office. With AVs, conducting activities while traveling is expected to occur much more often. The integration with the land use model SILO [21] will allow reflecting the impact of AVs on location housing choice. Some households might choose to move further away from their workplace, as commuting will become more convenient with AVs. Others might decide to move closer towards downtown, as lack of parking space could be eliminated with shared AVs or private AVs that park outside of town. Modeling the impact of AVs on household location choice and firm location choice will be a key improvement to reasonably assess travel demand in the future. In the long-run, it is intended to account for improved speed flow relationships of automated highways, modal shifts between existing and new transportation modes, changes in value of time when traveling in AVs, and induced travel demand.

\section{Data Availability}

SILO and MATSim codes are open source and available through the GitHub repositories https://github.com/msmobility/silo and https://github.com/matsim-org/matsim, respectively Road network was obtained from open data at https://www.openstreetmap.org. Due to privacy restrictions, survey results and synthetic population cannot be distributed.

\section{Conflicts of Interest}

The authors declare that there are no conflicts of interest regarding the publication of this paper.

\section{Acknowledgments}

The research was completed with the support of the Technische Universität München-Institute for Advanced Study, funded by the German Excellence Initiative and the European Union Seventh Framework Programme under Grant Agreement no. 291763. This work was supported by the German Research Foundation (DFG) and the Technical University of Munich (TUM) in the framework of the Open Access Publishing Program. The authors thank Matthias Fiedler, from the Department of Transport Planning and Mobility Management in the city of Fürstenfeldbruck, for his support on the distribution of the survey on the city website and the monthly magazine.

\section{References}

[1] D. Milakis, M. B. Snelder, B. Van Arem, G. Van Wee, Homem., and D. A. Correia, "Exploring Plausible Futures of Automated Vehicles in the Netherlands: Results from a Scenario Analysis," 
in Proceedings of the Automated Vehicles Symposium, San Francisco, 2015.

[2] A. T. Moreno, "Autonomous Vehicles: Implications on an Integrated Land-Use and Transport Modelling Suite," in Proceedings of the 11th AESOP Young Academics Conference, Munich, 2017.

[3] M. P. Enoch, "How a Rapid Modal Convergence into a Universal Automated Taxi Service Could Be the Future for Local Passenger Transport," Technology Analysis Strategic Management, vol. 27, no. 8, pp. 910-924, 2015.

[4] J. Bischoff and M. Maciejewski, "Autonomous Taxicabs in Berlin - A Spatiotemporal Analysis of Service Performance," Transport Research Procedia, vol. 19, pp. 176-186, 2016.

[5] D. J. Fagnant and K. M. Kockelman, "Dynamic ride-sharing and fleet sizing for a system of shared autonomous vehicles in Austin, Texas," Transportation, pp. 1-16, 2016.

[6] OECD, "Urban Mobility System Upgrade: How Shared SelfDriving Cars Could Change City Traffic," in Corporate Partnership Board Report, 2015.

[7] C. Llorca, A. T. Moreno, and R. Moeckel, "Effects of Shared Autonomous Vehicles on the Level of Service in the Greater Munich Metropolitan Area," in Proceedings of the mobil.TUM 2017, Munich, 2017.

[8] D. Milakis, M. Snelder, B. Van Arem, B. Van Wee, and G. H. De Almeida Correia, "Development and transport implications of automated vehicles in the Netherlands: Scenarios for 2030 and 2050," European Journal of Transport and Infrastructure Research, vol. 17, no. 1, pp. 63-85, 2017.

[9] M. Kümmel, F. Busch, and D. Z. W. Wang, "Framework for automated taxi operation: The family model," Transportation Research Procedia, vol. 22, pp. 529-540, 2016.

[10] H. Billhardt, A. M. Fernandez, S. Lujak et al., "Multi-Agent Systems and Agreement Technologies," in Proceedings of 13th European Conference, EUMAS, 2015, and Third International Conference, Athens, Greece, 2015.

[11] M. Ramezani and M. Nourinejad, "Dynamic modeling and control of taxi services in large-scale urban networks: A macroscopic approach," Transportation Research Procedia, vol. 23, pp. 41-60, 2017.

[12] W. E. Walker and V. A. Marchau, "Dynamic adaptive policymaking for the sustainable city: The case of automated taxis," International Journal of Transportation Science and Technology, vol. 6, no. 1, pp. 1-12, 2017.

[13] L. M. Martinez and J. M. Viegas, "Assessing the impacts of deploying a shared self-driving urban mobility system: An agent-based model applied to the city of Lisbon, Portugal," International Journal of Transportation Science and Technology, vol. 6, no. 1, pp. 13-27, 2017.

[14] J. Alonso-Mora, S. Samaranayake, A. Wallar, E. Frazzoli, and D. Rus, "On-demand high-capacity ride-sharing via dynamic trip-vehicle assignment," Proceedings of the National Acadamy of Sciences of the United States of America, vol. 114, no. 3, pp. 462-467, 2017.

[15] J. P. Zmud and I. N. Sener, "Towards an understanding of the travel behavior impact of autonomous vehicles," Transportation Research Procedia, vol. 25, pp. 2500-2519, 2017.

[16] P. Bansal, K. M. Kockelman, and A. Singh, "Assessing public opinions of and interest in new vehicle technologies: An Austin perspective," Transportation Research Part C: Emerging Technologies, vol. 67, pp. 1-14, 2016.

[17] R. Krueger, T. H. Rashidi, and J. M. Rose, "Preferences for shared autonomous vehicles," Transportation Research Part C: Emerging Technologies, vol. 69, pp. 343-355, 2016.
[18] C. J. Haboucha, R. Ishaq, and Y. Shiftan, "User preferences regarding autonomous vehicles," Transportation Research Part C: Emerging Technologies, vol. 78, no. Part C, pp. 37-49, 2017.

[19] F. Becker and K. Axhausen, "Literature review on behavioral experiments for autonomous vehicles," in Proceedings of the Annual Meeting of the Transportation Research Board, Washington, D.C., USA, 2017.

[20] J. Molloy and R. Moeckel, "Automated Design of Gradual Zone Systems. An iterative algorithm to design optimally sized spatial zones suitable for spatial modeling, while respecting municipal boundaries," Open Geospatial Data, Software and Standards, vol. 2, no. 19, pp. 1-10, 2017.

[21] R. Moeckel, "Constraints in household relocation: Modeling land-use/transport interactions that respect time and monetary budgets," Journal of Transport and Land Use, vol. 10, no. 1, pp. 211-228, 2013.

[22] A. T. Moreno and R. Moeckel, "Population synthesis handling three geographical resolutions," ISPRS International Journal of Geo-Information, vol. 7, no. 5, p. 174, 2018.

[23] R. Follmer, B. Lenz, P. Loecker, and M. Sigismund, in Proceedings of the Annual Meeting of the Transportation Research Board, Washington, D.C., USA, 2012.

[24] A. Horni, K. Nagel, and K. W. Axhausen, "The Multi-Agent Transport Simulation," in MATSim, Ubiquity Press, London, UK, 2016.

[25] M. Maciejewski, J. M. Salanova, J. Bischoff, and M. Estrada, "Large-scale microscopic simulation of taxi services. Berlin and Barcelona case studies," Journal of Ambient Intelligence and Humanized Computing, vol. 7, no. 3, pp. 385-393, 2016.

[26] Y. Croissant, "Estimation of Multinomial Logit Models in R: The Mlogit Packages: an Introductory Example," in Data Management, p. 73, 2003.

[27] R. Kitamura, S. Fujii, and E. I. Pas, "Time-use data, analysis and modeling: Toward the next generation of transportation planning methodologies," Transport Policy, vol. 4, no. 4, pp. 225-235, 1997.

[28] A. Moreno and R. Moeckel, "A Framework to Respect Travel Time Budgets as Constraint for Microscopic Destination Choice Modeling," in Proceedings of the the Annual Meeting of the Transportation Research Board, Washington, D.C., USA, 2017.

[29] A. Moreno, A. Michalski, C. Llorca, and R. Moeckel, "Autonomous Taxis Effect on Vehicle-Km Traveled and Average Trip Duration in the Greater Munich Metropolitan Area," in Proceedings of the the Annual Meeting of the Transportation Research Board, Washington, D.C., USA, 2018. 


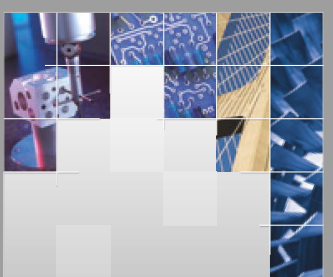

\section{Enfincering}
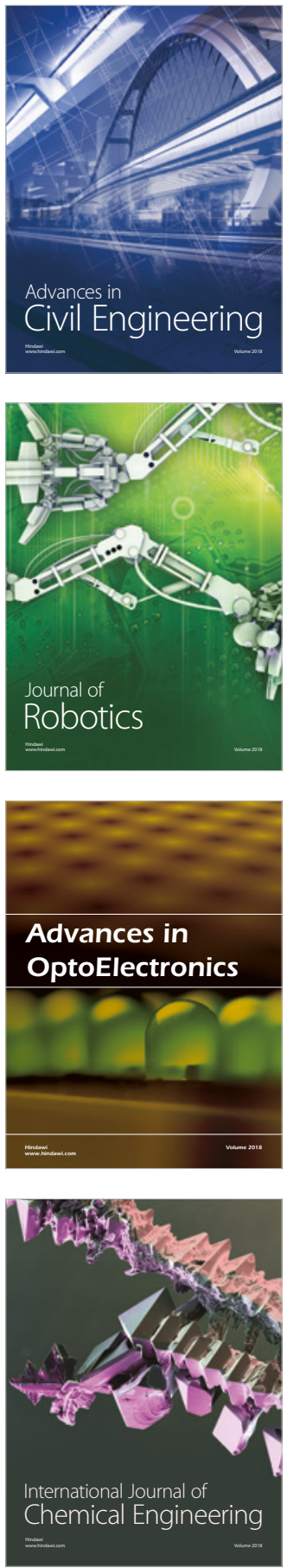

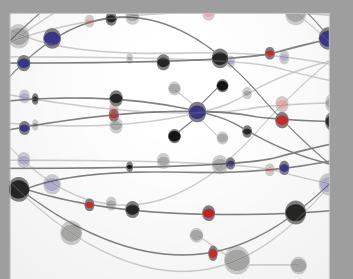

\section{Rotating \\ Machinery}

The Scientific World Journal

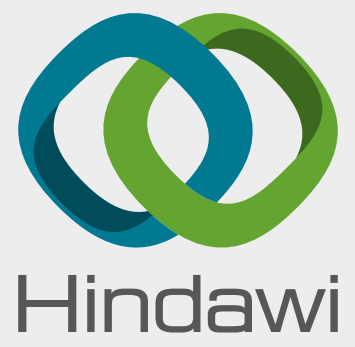

Submit your manuscripts at

www.hindawi.com
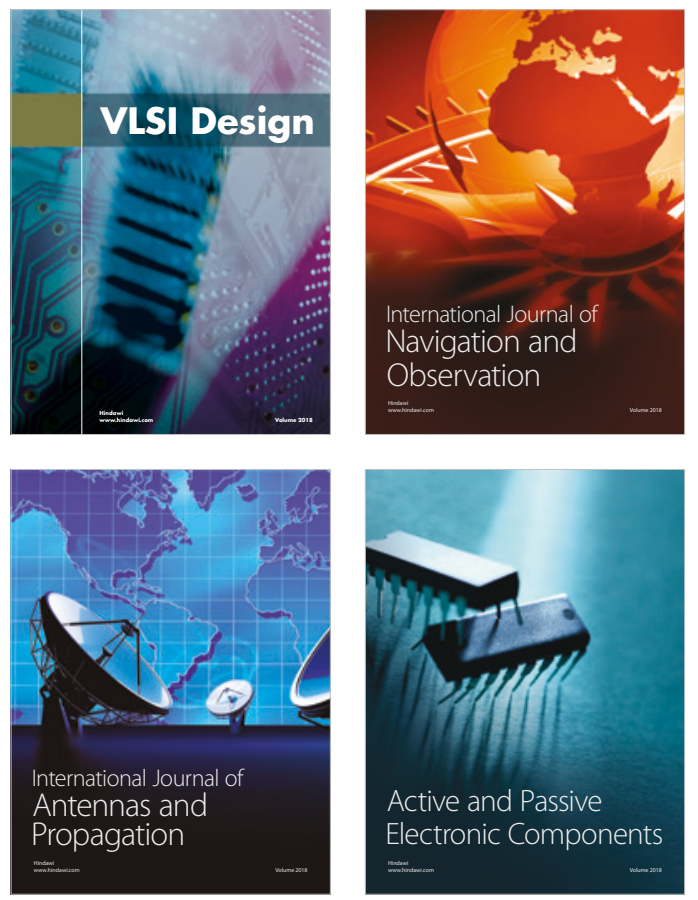
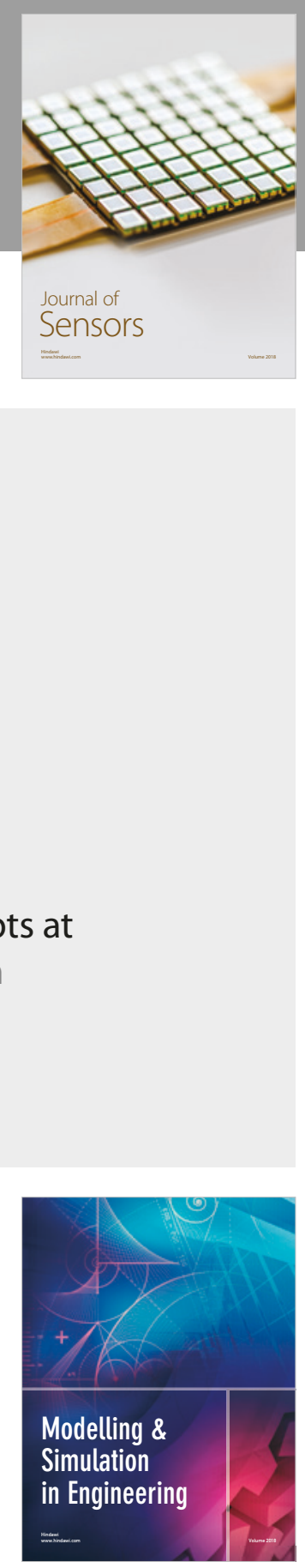

\section{Advances \\ Multimedia}
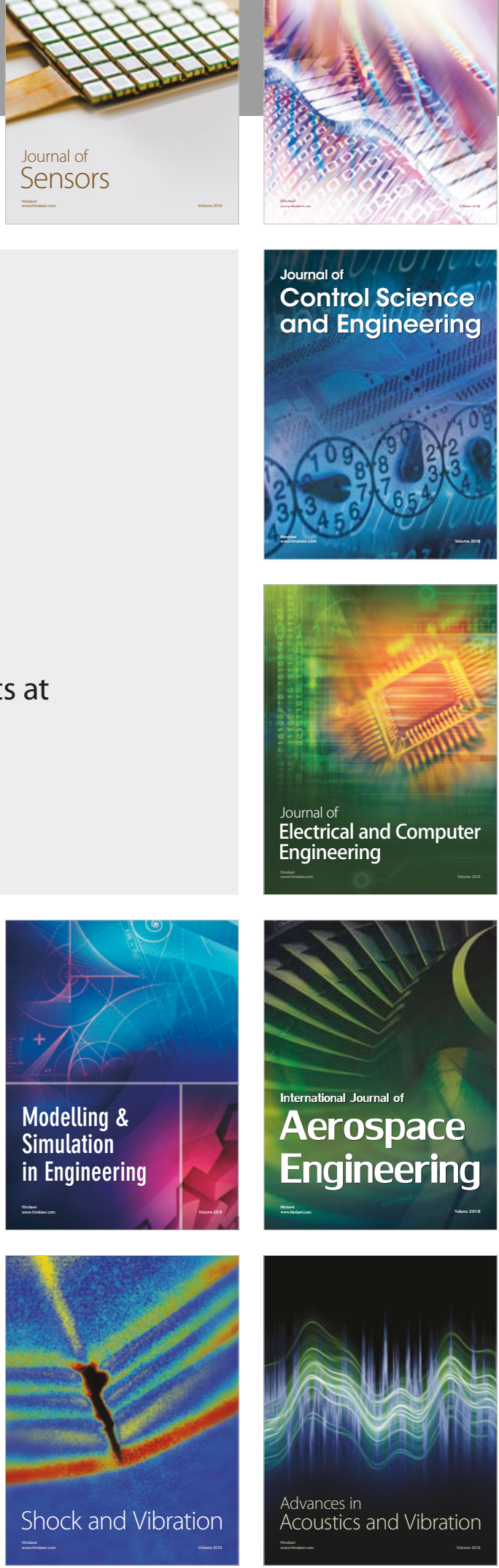